Cahiers de recherches médiévales

Journal of medieval studies

$11 \mid 2004$

Figures mythiques médiévales auX $\mathrm{XIX} \mathrm{X}^{\mathrm{e}}$ et $\mathrm{XX} \mathrm{X}^{\mathrm{e}}$ siècles

\title{
En guise de préface
}

\section{Alain Montandon}

\section{(2) OpenEdition}

Journals

Édition électronique

URL : https://journals.openedition.org/crm/1683

DOI : $10.4000 / \mathrm{crm} .1683$

ISSN : 1955-2424

Éditeur

Honoré Champion

Édition imprimée

Date de publication : 15 décembre 2004

Pagination : 7-17

ISSN : 1272-9752

Référence électronique

Alain Montandon, « En guise de préface », Cahiers de recherches médiévales [En ligne], 11 | 2004, mis en ligne le 06 mars 2008, consulté le 15 décembre 2022. URL : http://journals.openedition.org/crm/1683 ; DOI : https://doi.org/10.4000/crm.1683 


\title{
RM
}

\author{
Figures mythiques médiévales aux $\mathrm{XIX}^{\mathrm{e}}$ et $\mathrm{XX}^{\mathrm{e}}$ siècles \\ En guise de préface
}

L'un des enjeux de la recherche comparatiste en ce début de troisième millénaire reste plus que jamais l'étude des mythes littéraires dans leur genèse, leur pouvoir d'irradiation et de contamination, dans leur structure et les sens qu'ils impliquent ainsi que dans la diversité de leur réception. Pierre Brunel et son équipe de Centre de recherches en littérature comparée (CRLC) de l'Université Paris IVSorbonne ont ainsi pu concevoir des ouvrages fondamentaux, devenus très vites des outils classiques de référence incontournable. Le Dictionnaire des mythes littéraires, celui des mythes féminins, celui des mythes fantastiques ainsi que celui des mythes contemporains balayent ainsi un grand nombre de mythes litteraires et sont des instruments fondamentaux pour la recherche.

Le Centre de recherches sur les littératures modernes et contemporaines (CRLMC) de l'Université Blaise Pascal s'est quant à lui voué à la sociopoétique des mythes. Branche de la mythocritique, la sociopoétique envisage le mythe dans une perspective surtout historique et non seulement comme fond culturel. Le mythe n'est pas une structure stable, mais au contraire consiste en une série de variations, de tensions entre des éléments stables et des éléments qui varient sans cesse, qui se métamorphosent et sont modifiés. Une sociopoétique du mythe prend en considération non seulement cet ancrage, mais étudie comment dans une idéologie donnée, dans une époque donnée, dans un milieu socio-politique spécifique, dans un ensemble de représentations sociales et culturelles, un mythe vient à reparaître, continuer à vivre, se réécrire et s'activer. Le mythe est lié à son énonciation : c'est ce qui est raconté à un moment donné, dans des circonstances données. Or prendre en considération les positions de l'énonciation, c'est tenir compte de la poétique - au sens fort - du mythe, de l'énonciation comme phénomène littéraire. Un mythe n'existe pas en essence, c'est une histoire, un schème repris sans cesse différemment, pour rendre le réel intelligible et lui donner un sens. La sociopoétique se donne donc pour objet d'études la manière dont les représentations sociales (prises au sens large) à une époque articulent, génèrent et structurent le mythe. Une telle recherche ne comprend pas seulement une perspective diachronique, elle s'enrichit d'analyses synchroniques qui examinent le statut du système mythique à une époque donnée ${ }^{1}$.

Ainsi des recherches de ce type ont-elles été menées autour de figures mythiques telles que Psyché, Écho, Judas, Marie-Madeleine, Caïn, le Minotaure, Mélusine, autour de la diffusion géographique d'un mythe comme celui du «lait de la

\footnotetext{
${ }^{1}$ Voir les actes des colloques «Mythes de la décadence » ou «L'Avant-garde et les mythes » (Presses Universitaires Blaise Pascal, Clermont-Fd).
}

Cahiers de Recherches Médiévales (XII - XV s.), 11, 2004 


\section{Alain MONTANDON}

mort $»^{2}$, et enfin autour du statut du mythe à une époque donnée ( Rire des dieux », «Mythes de la décadence», «L'Avant-garde et les mythes», etc.)

La permanence et la rémanence de l'image médiévale est un phénomène des plus intéressants. L'image romantique du Moyen Âge et de ses mythes est bien connue et un ouvrage collectif, «La Fabrique du Moyen Âge», doit être publié aux éditions Champion. Le romantisme allemand avait le premier, dans son besoin de mythologies nouvelles, puisé dans le Moyen Âge un idéal, mais aussi tout particulièrement une imagerie que le néoromantisme a exacerbée dans un sens troubadour, celui de légendes aux tonalités mièvres ou dans le sens d'un sublime plus ou moins terrifiant et grandiose dont les mythes wagnériens seront l'une des expressions les plus marquées. Le Moyen Âge est moins une histoire que des histoires, moins un passé que la marque d'une antiquité qui perdure, moins un moment de l'histoire qu'une origine auquel on fait appel dès lors que le besoin d'un ressourcement se fait sentir. À titre d'exemple on pourrait citer le mythe de Mélusine, qui est bien chez Jean d'Arras un mythe des origines, un mythe fondateur. Celui-ci est intéressant non seulement parce qu'il témoigne de la richesse de l'imaginaire médiéval, mais en raison d'une fortune prospère attestant de la diversité de ses sens surdéterminés et de la variété de ses réécritures. Ceci s'explique peut-être d'abord par de l'image de l'identité féminine qu'il offre, l'image d'un mystère, d'une monstruosité et d'une part inappréhendable qui se dérobe à l'univers masculin (l'envol de Mélusine, son départ).

On peut naturellement voir dans cette histoire une rêverie autour du mystère du sexe féminin, y reconnaître le déni de la castration avec la fameuse queue de sirène, et bien d'autres éléments qu'une interprétation psychanalytique ne manquera pas de développer. Mais ce serait singulièrement limiter la dimension du mythe mélusinien, que d'en rester à de tels éléments premiers. Le mythe de Mélusine est intéressant en effet parce qu'il traverse, depuis le Moyen Âge, l'imaginaire collectif et qu'il se concrétise dans un certain nombre de réécritures jusqu'au $\mathrm{XX}^{\mathrm{e}}$ siècle. Il y a d'une part une histoire bien connue, faisant partie de l'imaginaire collectif, telle qu'elle a pu être véhiculée par exemple par la Bibliothèque bleue, histoire «bien connue» (le bien connu comporte cependant une grande part d'imprécision et de variantes!), réduite à des éléments majeurs, version sommaire si l'on peut dire. Et d'autre part un grand nombre de textes dans lesquels le mythe se déploie avec précision, détails, dans une richesse d'écriture qui en manifeste (que ce soit dans l'écriture littéraire, la peinture ou la musique) l'intense et fascinante présence. Ce dont parle le mythe c'est cette part de mystère irréductible, qui restera toujours chez l'homme dans sa représentation de l'autre. Sa fonction semble être l'inscription de la différence des sexes comme le non représentable, ce dont seul le mythe peut rendre compte.

En abordant le mythe dans la perspective d'une sociopoétique, c'est-à-dire de son émergence, dans les réécritures à partir de lectures nouvelles suivant des constellations socio-culturelles, politiques, historiques variées, nous avons quelques

${ }^{2}$ Le lait de la mort. La ballade de l'emmurée et sa fortune littéraire. Études réunies par Véronique Gély-Ghedira, "Cahiers de recherches du CRLMC », Clermont-Ferrand, 1998, 295 p. 
outils pour mieux comprendre l'évolution du mythe dont on va voir qu'il peut recouvrir des sens et des interprétations différentes, même si le mythe persiste en tant que tel avec certains mythèmes fondamentaux. Il y a à la fois permanence et mobilité. La ductilité du mythe, c'est sa capacité de donner forme à une série d'interrogations existentielles qu'il préfigure. Aussi une sociopoétique du mythe doit-elle permettre de suivre la vie du mythe Mélusinien à travers ses modalités historiques d'écriture : comment certains éléments dans une situation donnée deviennent-ils signifiants, comment certains traits prennent-ils le devant de la scène, ou comment certains autres s'estompent. Le mythe n'existe, ne s'accomplit, ne se déploie qu'à travers son histoire, qui est aussi celle d'une culture à laquelle, comme nous l'avons dit, il offre des schèmes d'intelligence.

Le mythe de Mélusine joue de la curiosité et de la peur du sexe féminin : le comte de Lusignan, transgressant le pacte conclu, épie sa femme un fatal samedi pour découvrir qu'elle a une énorme queue de poisson, qui lui inspire peur, dégoût, colère et rejet. Cette ambivalence de l'image féminine, qui engendre fascination émerveillée et répulsion horrifiée, comporte plusieurs aspects. Mélusine est à la fois une certaine forme de la nature : elle est fée de la nature, elle en incarne la fécondité, elle est mère nourricière, bâtisseuse et défricheuse. Cette position a pu être interprétée au départ comme représentation matriarcale, puisqu'elle est celle qui fonde la lignée, qui apporte la gloire, la richesse, la force, une interprétation que l'anthropologie contemporaine nie dans sa réalité, puisqu'elle affirme qu'il n'existe pas de société matriarcale. Mais le problème n'est pas là : nous nous intéressons à l'imaginaire et à ses représentations; nous voyons qu'il y a avec Mélusine l'image d'une femme originelle, qu'elle incarne comme femme et comme mère l'origine.

Cette imagination n'est pas corroborée par les textes dans la mesure où le destin de Mélusine chez Jean d'Arras comme chez Couldrette, c'est-à-dire dans la version médiévale, provient d'une transgression de la fille vis-à-vis de la loi du Père. La fille, Mélusine, a voulu venger sa mère et a subi un châtiment venant de sa propre mère (furieuse sans doute du fait que sa fille ait voulu prendre sa place) qui la condamne à cette monstruosité, celle d'un tabou périodique. Le mythe fonde ainsi la différence des sexes en exposant la féminité comme sexe dans son anatomie fantasmatique et sa physiologie.

À cette première impression stéréotypée d'une Mélusine matriarcale, se superpose l'image d'une société patriarcale, celle de l'époque médiévale, où l'homme est seigneur et maître et où l'espace réservé à la femme est une place spécifique. La femme apparaît comme celle qui va éduquer et maîtriser les passions absolument violentes de l'homme. Elle est bâtisseuse et défricheuse, mais surtout civilisatrice. Cette fonction est, en filigrane, une fonction menacée. La violence de l'homme va reprendre le dessus. Si Mélusine apporte ses pleurs de deuil et revient la nuit pour éduquer ses enfants, c'est qu'elle a été victime de cette violence.

On a donc dans le mythe médiéval, outre l'importante dimension économique de cette femme maternelle et défricheuse qui a été bien mise en valeur par Le Goff, un enjeu de société matriarcale/patriarcale, d'opposition et de conflit entre pouvoir féminin et pouvoir masculin. Les différentes versions et remaniements font apparaître une double figure, positive et négative, dont l'ambivalence sert de cadre à exprimer l'incertitude dans la relation à l'autre. Il conviendrait d'évoquer ici éga- 


\section{Alain MONTANDON}

lement le fait que Mélusine tient une place privilégiée dans un enjeu théologicoculturel, elle est le passage entre une mythologie païenne et la religion chrétienne.

La légende de Mélusine était populaire au XVIII ${ }^{\mathrm{e}}$ siècle grâce à la Bibliothèque Bleue. Des expressions passées à l'état quasi proverbial en témoignent: des histoires de Mélusine (pour parler de contes à dormir debout), des cris de Mélusine (pour désigner des cris perçants). Plus généralement, sylphes, ondins et autres esprits élémentaires qui nourrirent quantité de contes et de récits avaient trouvés dans Le Comte de Gabalis ou Entretiens sur les sciences secrètes de Montfaucon de Villars publié en 1670 à Paris une source d'inspiration évidente ${ }^{3}$. Montfaucon de Villars s'est inspiré en particulier du Liber de nymphis, sylphis, pygmaeis et salamandris de Paracelse qui fait place à la figure de Mélusine, présentant celle-ci sous un terme générique qui voit dans la fée une fille du diable:

Les Mélusines sont des filles de rois, désespérées à cause de leur péchés. Satan les enleva et les transforma en spectres, en esprits mains, en revenants horribles et monstres affreux. On pense qu'elles vivent sans âme raisonnable dans un corps fantastique, qu'elles se nourrissent des éléments et qu'au jugement dernier elles passeront avec eux, à moins qu'elles ne se marient avec un homme. Alors, par la vertu de cette union, elles peuvent mourir de mort naturelle, comme elles peuvent vivre naturellement dans le mariage. De ces spectres on croit qu'il y a plusieurs dans les déserts, les forêts, les ruines et les tombeaux, les voûtes vides et sur les bords de la mer.

La citation est importante, car elle fait de Mélusine un esprit qui aspire au mariage avec un être humain pour acquérir une âme (thème repris avec force par de nombreux auteurs et en particulier La Motte Fouqué dans son Ondine, pendant le romantisme allemand où la question de l'identité du sujet se pose avec acuité) et en même temps une figure spectrale, qui, n'arrivant pas à s'incarner, reste le fantôme d'un vide hantant les lieux désolés. L'ouvrage de Montfaucon qui connut un grand succès est ironique et critique sur le fond, mais fécond quant aux ressources de l'imagination, de la fantaisie et de la fiction. Ce qui est intéressant c'est que les esprits élémentaires sont une place vide prête à de nouvelles interprétations, pour désigner un au delà, une énigme, un ineffable qui ne peut se décrire, renvoyant à un passé archaïque qui prend la forme d'un Moyen Âge mythique auquel les auteurs se confrontent. Nous assistons à une réécriture de l'hypotexte dans de nouvelles perspectives qui mêlent l'irréalité à la scène historique.

La malice de la fée qui cherche un mari terrestre s'allie à un jeu érotique fort courant au XVIII ${ }^{\mathrm{e}}$ avec les sylphides séductrices et les sylphes invisibles qui sont des amants parfaits. Parmi la production fantasque et ambiguës de Marguerite de Lubert, La Princesse Camion est un conte particulièrement intéressant en ce qu'il se nourrit du thème mélusinien pour évoquer non sans grivoiserie l'acte de la défloration qui fait pousser à la princesse des cris de Mélusine. Ainsi le cri de Mélusine prend-il dans ce conte libertin du siècle des Lumières un sens très nouveau et précis qui est celui d'une perte heureuse et qui loin de séparer les amants les réunit pour

${ }^{3}$ Roger Laufer parle d' «influence limitée mais incontestable» (Le Comte de Gabalis ou Entretiens sur les sciences secrètes de Montfaucon de Villars avec une introduction et un commentaire de Roger Laufer, Nizet, Paris, 1963, p. 9). 
toujours dans le bonheur. Cette réécriture libre et ludique du thème mélusinien dans la tradition du conte de fée galant suit le hasard et le caprice d'une «raison irraisonnable [...] dans lequel le monstrueux se vit comme un passage, où chacun peut à loisir - et à plaisir-mesurer ce que soi-même et l'autre portent d'ensauvagement $»^{4}$. L'objet de désir et de répulsion doit être mis à mort dans une funèbre cérémonie qui dans le jeu d'ombres et de lumière ne cède en rien au merveilleux d'antan et aux terreurs médiévales.

Dans le récit de Goethe La Nouvelle Mélusine, Mélusine devient la plus charmante des petites naines. La réécriture du mythe subit de singuliers renversements : le chevalier n'est plus qu'un humain, sorte de jeune vagabond picaresque insouciant pour qui le pacte n'est qu'une entrave insupportable à sa liberté de célibataire. La nouvelle Mélusine, reine des richesses de la terre et des métaux, est une naine qui habite dans un coffret qui tient ici place de baignoire. La monstruosité signifie la dissymétrie dans la relation du couple et la relativité qui pèse sur l'existence humaine. Sous les transformations pleines de légèreté se dessine chez Goethe une profondeur lourde de sens. Il s'agit bien de mettre la femme en boîte et d'en terminer avec la puissance matriarcale, celle de la Mère Lusigne. Nous sommes dans un monde où le père de Mélusine retrouve ses droits. Le conflit entre le désir et le mariage, l'érotisme et la perpétuation de l'espèce est «résolu» en ce que Mélusine est amante sous une forme humaine et mère sous la forme minuscule. L'échec de la relation amoureuse signale l'aporie des rapports entre sexe qui est mis en scène suivant un mécanisme psychologique de défense.

Ce traitement du mythe de Mélusine nous éloigne de l'imaginaire original. Certes elle représente la beauté, la fécondité, la richesse, le merveilleux mais le héros, aventurier à la tête folle, épris de liberté et de vagabondage, n'y est guère sensible. Mélusine représente de manière ironique l'image du lien social, du mariage, dont le héros ne veut pas entendre parler.

Le romantisme allemand intègre la figure de Mélusine dans plusieurs textes. Brentano et Arnim en particulier lui consacrent des pages fort intéressantes qui confrontent Mélusine à la Révolution française.

Le premier texte inachevé ${ }^{5}$ de Brentano a été intitulé par la critique Raimondin. L'histoire ne se passe plus dans un légendaire Moyen Âge, mais à l'époque de la Révolution française qui voit la destruction par les flammes du bel arbre généalogique des Lusignan aux belles images. Car la légende, ce sont d'abord des images. C'est aussi l'importance du nom, du mot qui provoque émotions et associations. C'est l'histoire de l'enfance et de l'origine, à la fois personnelle et historique. Mélusine signifie la nostalgie du paradis perdu de l'enfance, qui est celui des belles images, de l'amour maternel auquel succèdent le divorce, la séparation. C'est aussi l'histoire de l'imagination enfantine qui se saisit de la légende, qui s'en déguise (le thème du déguisement y est important par la suite) et qui alimente la rêverie de l'enfant, c'est une histoire comme un rêve, où l'on dort, où l'on s'éveille, où l'enfant écoute et entend pendant la nuit les domestiques... C'est une histoire

${ }^{4}$ Elisabeth Lemirre, Postface à l'édition de La Princesse Camion, Mercure de France, 1995, pp. 86-87.

${ }^{5} \mathrm{Ce}$ fragment a été retrouvé en 1944 et publié par Friedrich Fuchs dans la Neue Rundschau (automne 1944). Il avait été écrit autour des années 1815-1816 (pendant l'hiver) à Berlin. 


\section{Alain MONTANDON}

œdipienne : le fils est condamnée par le Père à brûler et détruire le cœur de la mère. Cette histoire de la chute, de la disparition du paradis chez un enfant qui oscille sans cesse des pleurs à l'insouciance prend une tournure poétique merveilleuse et fantastique.

Tout homme est un Raimondin, aimant une fée qui le trahit et l'abandonne. Mais toutes les femmes apparaissent également comme des Mélusines. Il ne s'agit plus ici de l'histoire de la femme à queue de poisson, de curiosité, de pacte violé. Ce qui intéresse c'est l'origine, la frontière entre l'origine maternelle et la vie terrestre historique de l'homme, une frontière qui est source d'antagonismes et de souffrances. L'origine de Mélusine n'est pas cachée : elle descend des Lusignan. Le conflit est socio-politique, parce que le père est un révolutionnaire ardent. Là gît la transgression, non plus dans la trahison de ce qui doit rester caché, mais dans un acte d'émancipation par lequel l'homme se détache de ses liens naturels et sociaux dont il a hérité de et par ses origines. Cette violation de l'inné, de l'héréditaire par la violence de l'histoire est la source du récit. Cet antagonisme est visible lors du jour de l'anniversaire (date symbolique rappelant l'hérédité naturelle). ${ }^{6}$ Toute la famille est comme un gigantesque organisme naturel et biologique. Mais le père se révolte et tourne sa colère contre cet organisme végétal, naturel (auquel il oppose une création artificielle, l'arbre de la liberté). La bonne fée de la légende, fée de la mer est devenue la proie des flammes de l'histoire. La démarche romantique consiste à retrouver le sens de la légende, du mythe face à l'histoire qui est vécue comme chute et comme expulsion du paradis ${ }^{7}$, perte de l'innocence, mélange de catastrophes familiales et historiques. Le sacrificateur est lui-même sacrifié : Raimondin, aliéné, déguisé, s'exprimant dans une langue étrangère (le français), brûle aussi son propre cœur en s'émancipant du lien maternel.

L'un des traits caractéristiques du mythe mélusinien est sa présence cachée, sans que la figure soit explicitement nommée ${ }^{8}$. Ainsi dans la Fée aux miettes de Nodier, l'écrivain joue sur la coexistence de deux mondes opposés, l'un positif et réaliste, l'autre, royaume de l'imagination et du merveilleux mystique, comme le faisait par ailleurs Hoffmann dans son Conte le Vase d'or, où Serpentina possédait de nombreuses résonances mélusiniennes. On retrouve cette ductilité du mythe avec le syncrétisme mythologique de Gérard de Nerval qui s'empare à maintes reprises de la figure mélusinienne, souvent brouillée et amalgamée à d'autres personnages légendaires. Je n'aborde pas cet aspect du mythe qui nous entrainerait trop loin, mais qui est fort intéressant dans le cadre d'une sociopoétique: considérer comment un mythe s'allie à d'autres, attire autour de lui d'autres constellations par coalescence. Les Filles du Feu aurait eu pour titre premier «Mélusine ou les filles du

\footnotetext{
${ }^{6}$ Le lapsus qui fait que la date anniversaire soit aussi celle du mariage peut lancer l'interprète sur la voie d'une origine sexuelle. L'anniversaire du mariage est un jour funeste, comme si le féminin, objet d'amour, était dégradé dans la sexualité présentée comme un péché mortel.

${ }^{7}$ Thème chez Brentano. Voir Wolfgang Frühwald, Das verlorene Paradis. Zur Deutung der Herzlichen Zuueignung des Märchens Gockel, Hinkel und Gackeleia, Literaturwissenschaftl. Jahrb. N.F., 3, p. 113 sq. (sans mention naturellement de Mélusine).

${ }^{8}$ Henri Béhar, fondateur de la revue d'études surréalistes, Mélusine, constate que si le titre s'impose pour évoquer le surréalisme, Mélusine dans le surréalisme est une figure assez peu courante.
} 
feu », avant qu'il ne songe par la suite à celui de «Les Amours perdues», et qu'il ne finisse par les Filles $d u$ Feu. C'est que celles-ci, avec bien d'autres, sont des figures de la perte. Mélusine est à la fois l'image d'une femme idéalisée, inaccessible et perdue. Représentation de l'impossible, elle est espace magique du rêve, hanté du motif de la rencontre amoureuse, rencontre merveilleuse d'un chevalier et d'une fée dans un décor médiéval. Le décor étrange et imprécis d'un château féerique encadre le visage d'Adrienne et la belle blonde, mi-princesse mi-fée, chante « une des ces anciennes romances pleines de mélancolie et d'amour, qui racontent toujours les malheurs d'une princesse enfermée dans sa tour ". La tour du château évoque non seulement l'enfermement ( Mme de Montfort prisonnière dans sa tour $»^{10}$ ), mais aussi l'idée de la hauteur où se joue l'articulation du terrestre et du céleste, de la chute et de l'envol. Si El Desdichado se définit comme le prince d'Aquitaine à la tour abolie, il ne signifie pas seulement une rupture généalogique, la perte du nom, de l'identité de la race, mais également la perte de cette communication symbolisée par la tour entre les forces terrestres et célestes, la brisure du lien entre les deux parts d'une identité cosmique, ce qui l'amènera à poser la question de l'alternative : «Suis-je Amour ou Phébus?... Lusignan ou Biron?» L'interrogation de l'identité, qui était déjà un noyau central du mythe médiéval, est reprise par le poète.

On a beaucoup glosé sur cette appartenance mythique d'El Desdichado, à la famille des Lusignan. Raymondin a perdu Mélusine et le poète a perdu la femme aimée : ténébreux, veuf et inconsolé. Nouvel Orphée, il invoque Mélusine telle une nouvelle Eurydice, placée elle aussi sous le signe fatidique du serpent. Faire renaître la femme aimée et perdue, au moins grâce à cet habit tissé par les fées qu'est le rêve, celui d'une grotte où nage la sirène, tel est le sens de l'appel à Mélusine, cette muse poétique dont il écrit dans Promenades et Souvenirs : «La muse est entrée dans mon cœur comme une pythie, en jetant des cris de douleur: seulement ses derniers accents se sont adoucis à mesure qu'elle s'éloignait. Elle s'est détournée un instant et j'ai revu, comme en un mirage les traits adorés d'autrefois. » La fée a une fonction inspiratrice, en particulier par cette voix si chère qui s'est tue et que le chant ranime.

Pierre Brunel a bien remarqué que la fée était inséparable du génie du lieu : Mélusine et Manto (Mantoue), Mélusine et Lusignan et la Lorelei, femme intimement lié au paysage du Rhin. C'est que le corps de la fée devient paysage (comme dans une métamorphose ovidienne), se fond dans le paysage, s'y évanouit. Poisson, étoile, végétal, elle est de la nature l'antique légende. Tour à tour emblème de la maternité, figure de l'amante, personnage de mystère, la fée médiévale est l'image de l'amante dérobée, ce fantôme disparaissant "en jetant des cris plaintifs $»^{11}$.

La qualité musicale du nom n'a pas attendu Franz Hellens (qui fait de Mélusine la «mélodie des usines ») pour faire l'objet de maintes rêveries ${ }^{12}$. Cette musique est pour l'époque romantique l'écho d'un passé médiéval magique. Un mythe, c'est d'abord un nom propre et le nom de Mélusine en cela est pour les romantiques un chiffre, celui du passé perdu, source de nostalgie d'une époque et d'un rapport à la

${ }^{9}$ Nerval, Euvres, Pléiade, I, p. 245.

${ }^{10}$ Nerval, Euvres, Pléiade, I, p. 142.

${ }^{11}$ Nuits d'octobre, Autre rêve, XXV.

${ }^{12}$ «Wer Melusine heisst, sollte wissen, was Namen bedeuten. » (Fontane, Stechlin). 


\section{Alain MONTANDON}

nature que les contemporains ont oublié. Au nom de Mélusine est lié un certain rapport harmonieux avec la nature. Le roman de Karl Frenzel ${ }^{13}$ fait apparaître l'héroïne éponyme dans son amour de la nature et des roses du château où elle est employée. Avec sa chevelure brune, son visage d'une noblesse romaine, Mélusine est une grande lectrice douée d'une vive imagination. C'est aussi une révoltée, vive et insaisissable, finalement un peu sorcière. Son drame est la passion qui ne peut s'accomplir dans la société humaine où elle ne se sent pas à sa place. Elle ne se sent bien qu'au milieu des éléments, le vent, l'eau, l'orage qui ont une grandeur et un sublime qui répond à ses sentiments. Histoire d'une passion destructrice, où l'héroïne se suicide à la fin, rejoignant les eaux du lac, ce roman d'amour n'a qu'un intérêt médiocre, la psychologie y est sommaire, mais il est intéressant de voir comment le nom de Mélusine sert à situer un caractère capricieux et fantasque de jeune fille ambitieuse et amoureuse passionnée.

Avec beaucoup plus de talent, Fontane poursuit le rêve de Mélusine, dont l'image conventionnelle est une sorte de forme creuse qui permet de structurer le réel sans cependant le conceptualiser. On peut dire que le motif traverse toute son œuvre, pour, par exemple, ironiser sur la noblesse à laquelle il trouve quelque chose d'amphibie, de non-humain. Le nom de Mélusine désigne pour Fontane l'absence d'âme comme vide psychique. La Mélusine moderne, Ozeane, mène dans un monde cosmopolite une vie sans sentiment ni sensibilité. Fontane tout en accentuant des éléments renvoyant au mythe traditionnel, utilise le nom par analogie et allusion. Ainsi une figure traditionnelle est intégrée à un discours romanesque moderne par intertextualité allusive pour créer un système d'échos permettant de mieux saisir la situation des personnages.

La pièce de Grillparzer, créée en 1823, conçue pour un opéra dont Beethoven devait composer la musique, représente la fée aspirant à l'union avec un humain. Mélusine est devenue elle-même une idée, l'objet d'une quête intérieure de la part du chasseur. Elle-même aspire à prendre forme et à connaître la chaleur des bras d'un homme. Mais l'idée pure qu'elle représente et la vie semblent inconciliables. L'espace de pure beauté n'est pas vivable. Et le héros, figure de l'artiste, découvrant la monstruosité dont est victime celle qui s'incarne par amour, sera pris d'une hésitation fatale, car il faut s'engager sans retour pour gagner l'art qui est une force supérieure à la vie et qui exige de renoncer au monde. Mélusine symbolise l'Art, une Idée, un Idéal, une potentialité («das Mögliche») : «Le rêve nous entoure, nous qui sommes le rêve». Identifiant donc Mélusine au possible et au rêve, Grillparzer met l'accent sur l'infini et l'atemporalité d'une telle Idée en opposition avec le caractère fini, achevé et matériel de l'existence. Cela signifie que l'Art est séparé des mortels. Ce n'est que dans l'union avec le réel qu'il peut se manifester. Mais l'art se dégrade dans ses manifestations. L'artiste doit renoncer au monde, car il n'y a pas de demi-mesure dans la dévotion: telle est la tragédie de l'artiste, le malheur d'être poète, d'occuper une position impossible entre l'Art et la Vie et seule la mort est la solution du dilemme.

Avec le décadentisme, et même avec Zola chez lequel Mélusine investit Nana, elle devient femme fatale, sphinge préraphaélienne, mêlant de manière oxymorique la nature la plus crue à l'artificialité la plus extrême, monstrueuse de silence

${ }^{13}$ Breslau, Verlag von Eduard Trewendt, 1860, 308 p. 
et d'innomé. L'aspect reptilien n'est pas absent pour se prêter à la constitution de l'image très forte de la femme fatale dont l'aura médiévale contribue à démoniser, avec plus ou moins de force, l'attrait. Le mythe permet à la fois un portrait assez misogyne de la femme, goule tyrannique et capricieuse, inspirant le désir sans l'éprouver elle-même, si ce n'est dans la contemplation narcissique de son propre corps ou dans des amours saphiques, et une critique de la société dont le comportement de Nana permet de dévoiler les vices et les stupres. Par un processus de divinisation et de décomposition, Mélusine s'inscrit dans un processus de thanatisation. Elle n'est plus figure rédemptrice, mais idole païenne offerte aux yeux de tous les hommes. Chez Jean Lorrain qui cherche dans les contes à retrouver les émotions délicates du merveilleux, Mélusine est princesse d'ivoire et d'ivresse propre à ourdir les belles tapisseries du songe. «Le charme est dans le beauté qui sommeille en la gaine squameuse et bruissante de l'hydre; la délivrance est dans le baiser du héros à l'âme assez trempée pour boire le poison et affronter la mort». Méduse, séductrice diabolique, elle prend à la fin du siècle des aspects monstrueux et tératologiques, telle la Mélusine de Péladan qui en fait une femme blessée à l'infirmité honteuse.

Avec Franz Hellens Mélusine est image d'une liberté fondamentale, irréductible qui caractérise son essence. Figure énigmatique hantée par un vertige d'interprétation qui n'est peut-être que le vertige même de la féminité inaccessible. Elle disparaît derrière sa blancheur, devient le «qui suis-je?» de ceux qui l'interrogent et, froide comme le silence, elle donne des signes à travers des parfums et des lumières que déchiffrent son amant et l'ingénieur Nilrem, réincarnation de Merlin, qui provoquera le dénouement de cette féerie en détruisant la cathédrale et en ravissant Mélusine.

Mélusine ne cesse de courir: elle est le rêve d'une verticalité, d'un envol, d'une libération. Déliée de la pesanteur, elle incarne l'ailleurs et la transcendance. Elle est lumière et mouvement, «faite pour la joie qui avance et la gaieté qui respire». Elle marche la première, selon son habitude. Ses pieds nus glissent sur le sable, elle porte une robe de saphir. Ses bras sont deux clartés de marbre qui ondulent. Son pas, sa légèreté, sa grâce, la vitesse de ses pieds nus sur le sol la caractérisent, tout comme son rire lumineux et son impertinence. Comme dans les tableaux de Delvaux, elle se promène nue, heureuse de marcher, cette nudité étant le signe de sa complète liberté, d'une délivrance de toutes les entraves.

Sa connivence avec la nature et les éléments signent son origine : particulièrement avec l'eau qui rappelle son origine aquatique et dont les métaphores prolifèrent et contaminent l'ensemble du texte. Mais il n'y a pas que le bas de sa robe qui trempe dans la mer: elle prend aussi des bains d'étoiles. Car elle est lumineuse et nocturne à la fois. La scène de la conversation avec Mélusine dans la salle de bain d'un hôtel (chapitre XIX) est le grand moment de la réécriture moderne de la fameuse scène médiévale au cours de laquelle Raymondin a épié sa femme dans sa baignoire un fatal samedi. Mais ici point de transgression - et c'est là la faute, l'erreur du héros : point de transgression, point de liberté. Quel dommage, car à Mélusine aux seins mouillés, aux courbes tendres, au corps blanc partagé à hauteur de ceinture par la surface de l'eau («Le buste tranché de Mélusine flottait sur l'eau, ses cheveux étaient sombres et ses bras étendus ressemblaient à deux avirons immergés à demi ») ne répond que l'indifférence immature du narrateur, qui cristallise 


\section{Alain MONTANDON}

les figures masculines, faibles et désemparées devant cette femme insaisissable. Mélusine inscrit le monde moderne dans son parcours : elle y croise le music-hall, le cinéma (avec la présence de Charlot), la peinture cubiste, l'art moderne le jazz, la vitesse, le machinisme, etc. Sa présence légère se dessine sur ce fond de vie contemporaine.

Pour Breton, dans Arcane 17, elle est également image de la libération, libération de l'humanité par l'essence féminine. Breton procède par glissements, de Méduse à Mélisande et de Mélisande à Mélusine, avec des effets de proximités sonores que Pierre Brunel a pu relever. Ce qui est ici significatif, c'est le mouvement de refoulement et d'idéalisation opéré. Méduse reste une menace pour Elisa qui est une rescapée du deuil (elle a perdu sa fille et a tenté de se suicider). Breton, rival de Méduse, sauve Elisa par le regard de son amour à l'occasion d'un voyage en Gaspésie: les cheveux de Méduse sont métamorphosés en cheveux de Mélisande qui elle-même laisse place à Mélusine dont le nom apparaît dans la deuxième partie d'Arcane 17. Pourquoi Mélusine? parce qu'elle est d'abord la mère, le cri de la mère (c'est le second cri de Mélusine, celui du retour) qu'il entend aussi lorsque les femmes disent adieu à la gare à leur mari qui partent à la guerre. Le cri de Mélusine est renversée par Breton, il n'est pas adressée au solitaire à la tour abolie, mais dans le retournement qu'il opère de Nerval («Ma seule étoile vit...»), il annonce non pas la mort, mais une nouvelle naissance. «Le second cri de Mélusine, [...] ce doit être le rêve de l'enfantement sans la douleur."

Breton fait de Mélusine (ou Mellusigne comme on l'écrivait parfois autrefois) la mère des signes. Celle par qui est donné à lire le réel. C'est elle qui renverse les signes pour un renouveau dans lequel la femme devient celle par qui le monde sera sauvé. Mélusine est serpent, elle reste l'ondoyante serpentiforme, la «torsade éblouissante», la courbe «qui ondule par vagues». Elle est dans le retournement que veut lui imprimer le poète, la femme serpent qui a vaincu le serpent, dont la lumière terrasse l'autre serpent, le Boa, «l'unique artisan de l'opacité». «Cette femme [...] qui est maintenant toute la femme» est opposée à la nonnommée, Lilith et toutes les figures de la femme maléfique et fatale androgyne, Lilith, la plaie, la cicatrice, à l'origine de cette grande malédiction qui est la dissociation du masculin et du féminin et qu'il s'agit de lever dans le rêve d'unité première («la grande malédiction est levée »). Le recours au mythe de Mélusine pour cet appel à l'image d'une femme qui en bannit les terreurs en redonnant vie à l'univers entier sera la consolatrice de celui qui amèrement se souvient de toutes les séparations et de toutes les ruptures.

Nous savons bien que nous n'avons fait qu'esquisser un survol rapide de quelques réécritures de ce mythe littéraire. Ce que je voudrais retenir, ce sont des pistes d'exploration et une perspective de recherches. Le mythe recèle une complexité qui n'apparaît pas au premier abord: la Mélusine médiévale est sans doute d'abord l'histoire de la fondation merveilleuse d'une famille, mythe fondateur d'une prospérité dont l'aspect économique est présent. C'est aussi une confrontation du masculin et du féminin sous de multiples aspects. C'est également le lieu d'un débat entre plusieurs cultures, culture païenne et culture chrétienne. Mais le mythe devient également l'espace d'un questionnement entre Histoire, tradition et rénovation, posant le problème de la liberté et de l'émancipation comme celui de la nature et de la civilisation. Ces thèmes sont diversement repris au fil des siècles 
suivant les perspectives de l'époque, non seulement suivant les conceptions politiques, mais également suivant les diverses représentations de la femme, servant tout à tour la misogynie la plus accentuée, comme l'idéalisation de la femme (ce qui n'est pas contradictoire avec la première position) ou à son émancipation libératrice.

Finalement le nom même de Mélusine finit par désigner des femmes au destin tragique qui ne peuvent s'insérer dans la société ni connaître une union harmonieuse. Elles retournent à la nature dont elles apprécient l'aspect sauvage. Ce tragique n'est pas sans s'unir à une part de monstruosité qui fait l'originalité et aussi le charme de la figure en ce qu'elle signe son caractère d'exception. La constellation mythique fait apparaître Mélusine comme une femme toujours désirée, inaccessible et qui finit par disparaître, par s'envoler dans les airs : elle est celle qui échappe toujours. Du coup le mythe inscrit un manque, une absence, un défaut, une rupture de communication. Le mythe parle de l'absence, de la rupture, du manque d'harmonie dans la relation humaine de la fée/femme. Nombreux sont les exemples dans la réécriture du mythe où l'on voit réapparaître cette absence de communication qui prend des sens différents, pas seulement dans le rapport à la sexualité, à la différence des sexes, mais aussi dans celui à la liberté, incarnée par cette femme qui justement, parce qu'elle conserve toujours en elle une part de secret, de mystère, échappe.

On pourrait prendre bien sûr d'autres exemples et penser à la belle étude réalisée par Isabelle Cani- Wanegffelen, Le Graal en question. Un mythe pour sortir de la modernité («Bibliothèque de l'Hermétisme», Dervy, Paris, 2003), à celle d'Isabelle Durand-Le Guern, Le Moyen Âge des romantiques (Presses Universitaires de Rennes, 2001) qui analyse entre autres les grandes figures mythiques de Charlemagne, Louis XI, Jeanne d'Arc, aux études d'Arlette Bouloumié (et en particulier à ce qu'un romancier comme Michel Tournier fait des mythes médiévaux). Ce numéro se situe dans la lignée de celui que les mêmes Cahiers de Recherches Médiévales avaient autrefois publiés en $1996\left(\mathrm{n}^{\circ} 2\right)$ et intitulé «Regards sur le Moyen Âge » qui, outre des perspectives plus générales et historiques s'était attaché également pour partie à quelques figures mythiques médiévales, celles par exemple de Clovis, de Robert le diable ou de Lancelot revu par Cocteau. La présente livraison apportera à ces études, nous l'espérons, des analyses et des perspectives nouvelles en cette période de troisième millénaire qui continue d'être, ô combien! fasciné par le Moyen Âge et ses grandes figures.

\author{
Alain Montandon \\ Université Blaise Pascal-CRLMC
}

